\title{
Métodos de pesquisa para o estabelecimento da eficácia das psicoterapias
}

\author{
Jan Luiz Leonardi
}

\section{RESUMO}

Em 2006, a American Psychological Association definiu o conceito de prática baseada em evidências em Psicologia como um processo individualizado de tomada de decisão clínica que ocorre por meio da integração entre a melhor evidência disponível com a perícia clínica no contexto das características, cultura e preferências do cliente. Entretanto, há pouco consenso na literatura sobre o que constituem evidências de boa qualidade, quais métodos deveriam ser empregados para produzi-las e a maneira pela qual elas deveriam ser aplicadas na prática do psicólogo clínico. Em vista disso, o presente artigo tem por objetivo apresentar os três métodos de pesquisa empírica que são apropriados para investigar a eficácia de intervenções psicoterápicas (ensaio clínico randomizado, experimento de caso único e estudo de caso), ressaltando os alcances e limites de cada um deles. Espera-se que este artigo possa contribuir para a formação de pesquisadores clínicos brasileiros e, por conseguinte, favorecer uma maior participação da Psicologia brasileira no movimento da prática baseada em evidências em Psicologia.

Palavras-chave: prática baseada em evidências; psicologia baseada em evidências; ensaio clínico randomizado; experimento de caso único; estudo de caso.

\section{ABSTRACT}

\section{Research methods for the establishment of the efficacy of psychotherapies}

In 2006, the American Psychological Association defined the concept of evidence-based practice in Psychology as an individualized process of clinical decision-making that occurs through the integration of the best available evidence with clinical expertise in the context of the characteristics, culture and preferences of the patient. However, there is little consensus in the literature about what constitutes good evidence, what methods should be employed to produce them and the way they should be applied in the practice of the clinical psychologist. As a result, this article aims to present the three methods of empirical research that are appropriate to investigate the efficacy of psychotherapeutic interventions (randomized clinical trial, singlecase experimental design and case study), highlighting the scope and limits of each of them. It is hoped that this article contributes to the formation of Brazilian clinical researchers and therefore advance a greater participation of Brazilian Psychology in the movement of evidencebased practice in Psychology..

Keywords: evidence-based practice; evidence-based psychology; randomized clinical trial; single-case experimental design; case study.

Há uma crescente preocupação entre os profissionais da área da saúde de que suas intervenções estejam solidamente sustentadas por evidências empíricas. Esse movimento, denominado prática baseada em evidências, foi desenvolvido originalmente na década de 1990 na Medicina e vem recebendo cada vez mais interesse por parte da Psicologia (e.g., Norcross, Beutler, \& Levant, 2006).

Após um longo embate entre diversas perspectivas teóricas, conceituais, metodológicas e

\section{Sobre o Autor}

J. L. L.

orcid.org/0000-0002-4166-4909

Universidade de São Paulo

(USP) - São Paulo, SP

janleonardi@gmail.com

\section{Direitos Autorais}

Este é um artigo de acesso aberto e pode ser reproduzido livremente, distribuído,

transmitido ou modificado, por qualquer pessoa desde que usado sem fins comerciais. 0 trabalho é disponibilizado sob a licença Creative Commons CCBY-NC. 


\section{WIINTERACÃO EM ET. PSICOLOGIA}

práticas sobre o assunto (Leonardi \& Meyer, 2015), a American Psychological Association (2006) definiu prática baseada em evidências em psicologia como um processo individualizado de tomada de decisão clínica que ocorre por meio da integração da melhor evidência disponível com a perícia clínica no contexto das características, cultura e preferências do cliente. Nessa perspectiva, os três elementos da definição evidências empíricas, repertório profissional e idiossincrasias do paciente - são fundamentais na tomada de decisão clínica, de modo que uma prática profissional não será considerada representativa desse modelo caso não considere a inter-relação entre os três componentes.

Entretanto, há pouco consenso na literatura sobre o que constituem evidências de boa qualidade, quais métodos deveriam ser empregados para produzi-las e a maneira pela qual elas deveriam ser aplicadas na prática do psicólogo clínico (Reed, Kihlstrom, \& Messer, 2006; Stiles et al., 2006). Por exemplo, alguns autores (e.g., Hollon, 2006) argumentam que o ensaio clínico randomizado é o melhor e, talvez, o único método apropriado para estabelecer a eficácia de uma psicoterapia, enquanto outros autores (cf. Persons \& Silberschatz, 2003) defendem exatamente o contrário, ou seja, que os resultados oriundos de ensaios clínicos randomizados são inúteis para a prática da Psicologia clínica.

Em suma, é possível encontrar diferentes pontos de vista na literatura (e. g. Reed, Kihlstrom, \& Messer, 2006; Stiles et al., 2006) sobre o valor dos diferentes métodos de pesquisa que visam estabelecer ${ }^{1}$ a eficácia de uma psicoterapia, de modo que um leitor que está adentrando o paradigma da prática baseada em evidências em Psicologia pode ficar perdido ou, pior, ter a impressão de que todo e qualquer dado conta como evidência. Em vista disso, este artigo tem por objetivo apresentar os métodos de pesquisa que são apropriados para investigar a eficácia das psicoterapias (ensaio clínico randomizado, experimento de caso único e estudo de caso) e realizar uma síntese dos diferentes pontos de vista sobre os alcances e limites de cada um desses métodos, visando oferecer um panorama mais imparcial sobre o assunto.

\section{ENSAIO CLÍNICO RANDOMIZADO}

O ensaio clínico randomizado, tipicamente, consiste na seleção de uma amostra de pacientes com um mesmo quadro clínico que são alocados randomicamente em dois grupos - um grupo experimental (no qual o tratamento em questão está presente) e um grupo controle (em que o tratamento em questão está ausente). Para evitar vieses, é fundamental que as pessoas envolvidas na pesquisa pesquisadores, cuidadores e os próprios participantes - não saibam a qual grupo pertencem, o que é chamado de cegamento. Para avaliar os resultados da exposição ao tratamento em questão, uma ou mais medidas quantitativas são realizadas com cada um dos participantes. Por fim, os dados obtidos são sumarizados por meio de testes estatísticos, o que possibilita afirmar se, na média, o tratamento é eficaz (Kendall, 2003). Deve-se observar que um ensaio clínico randomizado pode comparar a eficácia de um tratamento em relação à ausência de tratamento (e.g., fluoxetina vs. placebo para depressão) ou comparar diferentes tratamentos entre si (e.g., fluoxetina vs. sertralina para depressão).

Na Medicina, o ensaio clínico randomizado é considerado o padrão-ouro para o estabelecimento da eficácia de diferentes tratamentos (e.g., farmacológicos), uma vez que esse método possibilita determinar com bastante precisão a relação de dependência entre as variáveis independente e dependente. Com o intuito de alcançar na Psicologia o mesmo rigor metodológico da Medicina, Rosenthal e Frank sugeriram, em 1956, que o ensaio clínico randomizado deveria ser utilizado na pesquisa em psicoterapia e, desde então, centenas de estudos empregando esse delineamento foram conduzidos. Nas últimas décadas, o ensaio clínico randomizado passou a ser visto por muitos pesquisadores (e.g., Hollon, 2006; Kendall, Comer, \& Chow, 2013) como o delineamento mais rigoroso para avaliar os resultados de determinada modalidade de psicoterapia e, por essa razão, tem sido utilizado para identificar as melhores intervenções terapêuticas para diferentes transtornos psiquiátricos.

No campo da psicoterapia, o ensaio clínico randomizado assume algumas características particulares. A amostra geralmente é composta por indivíduos com uma psicopatologia listada no Manual Diagnóstico e Estatístico dos Transtornos Mentais (DSM; American Psychiatric Association, 2013) e aqueles que apresentam comorbidades muitas vezes são excluídos da pesquisa. Os participantes são distribuídos aleatoriamente entre os grupos experimental e controle, sendo que a intervenção consiste na aplicação de procedimentos padronizados descritos num manual. 0 uso dos manuais de tratamento é fundamental para indicar com precisão os procedimentos terapêuticos envolvidos (i.e., a variável independente) e diminuir as idiossincrasias dos terapeutas envolvidos. Para garantir a fidedignidade da intervenção, os terapeutas são previamente treinados a seguir o manual e, ao longo da pesquisa, a correspondência entre seu desempenho e as prescrições dos manuais é monitorada. Os participantes são avaliados antes e depois do período de intervenção por meio de medidas de autorrelato (e.g., Inventário de Depressão de Beck) ou outras modalidades de mensuração de resultado (e.g., frequência de um comportamento-alvo), e os desfechos são analisados estatisticamente (Starcevic, 2003). De modo semelhante à pesquisa farmacológica, um ensaio clínico randomizado pode comparar a eficácia de determinada psicoterapia em relação à sua ausência (e.g., terapia cog- 


\section{-3: INTERACÃO EM LF PSICOLOGIA}

nitiva vs. placebo para depressão), comparar diferentes formas de terapia entre si (e.g., terapia cognitiva vs. psicanálise para depressão) ou compará-la com um tratamento farmacológico (e.g., terapia cognitiva vs. fluoxetina para depressão).

Apesar de suas potencialidades, o uso do ensaio clínico randomizado na pesquisa em psicoterapia recebeu muitas críticas. Uma delas é que definir os problemas clínicos pelo DSM restringe as problemáticas pesquisadas, excluindo do escopo de investigação os pacientes cuja queixa clínica não é contemplada pela semiologia psiquiátrica (Starcevic, 2003). De modo semelhante, o uso de manuais de tratamento é criticado por serem elaborados para transtornos específicos, o que limitaria os tópicos de pesquisa e dificultaria a necessária adaptação do repertório do terapeuta às singularidades de cada cliente (Nathan, Stuart, \& Dolan, 2000).

Algumas das críticas ao uso do ensaio clínico randomizado na pesquisa em psicoterapia referem-se a limitações metodológicas impostas por características intrínsecas à psicoterapia. Um exemplo é a impossibilidade de estabelecer o cegamento: o terapeuta invariavelmente sabe qual é a intervenção que está oferecendo e os participantes podem vir a ter algum conhecimento do tratamento que estão recebendo. Outro exemplo diz respeito à padronização da intervenção que está sendo investigada (i.e., da variável independente em questão). Na pesquisa farmacológica, todos os participantes recebem exatamente a mesma dose do mesmo medicamento, o que é impossível de ser reproduzido na pesquisa psicológica. Em primeiro lugar, o conjunto de ações que um terapeuta deve executar ao longo de um processo psicoterápico é enorme e variado, de modo incomparável à administração de um comprimido. Além disso, mesmo que previstos e bem descritos num manual, os procedimentos terapêuticos raramente podem ser aplicados da mesma forma em todos os casos, pois cada sessão com cada cliente tem especificidades que não são passíveis de serem previstas, controladas ou padronizadas (Starcevic, 2003). Novamente, é fundamental ressaltar que, embora os críticos ao ensaio clínico randomizado apontem a impossibilidade de estabelecer o cegamento e a padronização da intervenção, tais limites metodológicos são inerentes à própria pesquisa em psicoterapia e, portanto, também estão presentes nos outros métodos de pesquisa, apresentados adiante.

Outra fonte de críticas ao ensaio clínico randomizado provém da composição dos grupos-controle. Muitas pesquisas comparam os resultados promovidos por determinada intervenção com os desfechos dos indivíduos que ficaram na lista de espera pelo tratamento. Neste tipo de estudo, o grupocontrole não passa por uma condição placebo e geralmente é informado que provavelmente não terá nenhuma melhora (Nathan, Stuart, \& Dolan, 2000). De acordo com Kihlstrom
(2006), comparar os efeitos de uma intervenção com a ausência de tratamento tem relevância limitada para o estabelecimento de eficácia, pois "é alguma coisa provar que a psicoterapia é melhor do que nada, mas certamente não é muito" (p. 27).

Outras pesquisas empregam um placebo psicológico para analisar os efeitos da intervenção. Nestes casos, é oferecido ao grupo-controle um tratamento com pouco ou nenhum benefício que, para ser plausível para o cliente, contém fatores comuns a todas as psicoterapias (e.g., empatia), mas não contém os procedimentos específicos que estão sendo investigados. Por essa razão, alguns autores (e.g., Baskin, Tiemey, Minami, \& Wampold, 2003) criticam a concepção de placebo psicológico, argumentando que tais tratamentos "dissimulados" não seriam inertes, uma vez que contêm fatores comuns que podem levar a mudanças clinicamente significativas (o que os tornariam tratamentos verdadeiramente ativos). Além disso, esses autores apontam também que o fato de o terapeuta saber que está oferecendo uma intervenção planejada para falhar pode comprometer seu envolvimento, de tal forma que as diferenças encontradas nos resultados do grupo experimental e do grupo controle poderiam ser fruto de seu baixo engajamento. Além disso, na maioria dos estudos (e.g. Baskin et al., 2003) o placebo psicológico não é estruturalmente equivalente ao tratamento em investigação: o grupo-controle passa por um número menor de sessões do que o grupo experimental; o formato de intervenção é diferente (terapia em grupo no grupo-controle e terapia individual no grupo experimental); os terapeutas do grupo-controle recebem menos treinamento e supervisão; etc. Assim, a superioridade de determinada intervenção poderia ser atribuída à disparidade da estrutura da intervenção e não às suas características.

Além da comparação da intervenção com lista de espera e placebo, alguns trabalhos têm como grupo-controle a intervenção que é geralmente oferecida por terapeutas com experiência no transtorno em questão, o que é chamado de tratamento usual. Portanto, este tipo de controle é apropriado para avaliar se a intervenção em investigação é melhor do que a que já vem sendo oferecida na comunidade. De acordo com Nathan et al. (2000), a comparação com o tratamento usual tem dois problemas. Um deles é que o tratamento usual não pode ser considerado um placebo genuíno, pois contém fatores comuns e, ainda, pode conter fatores específicos da intervenção em investigação, comprometendo a validade interna da pesquisa. 0 outro é que demonstrar igualdade ou superioridade de uma nova intervenção em relação ao tratamento usual pode ser irrelevante caso ele não tenha eficácia comprovada.

Para alguns autores (e.g., Borkovec \& Castonguay, 2006; 


\section{- M. INTERACÃO EM LF PSICOLOGIA}

Starcevic, 2003), a validade interna do ensaio clínico randomizado - garantida pela homogeneidade diagnóstica dos participantes, delimitação do número de sessões e protocolo de atendimento manualizado - pode comprometer a generalização dos resultados para a prática cotidiana da psicoterapia (validade externa). Nesse sentido, Persons e Silberschatz (2003) argumentam que o cliente típico do consultório é exatamente o que tem maior probabilidade de ser excluído de um ensaio clínico randomizado e que a rigidez concernente ao tempo de duração da terapia e à uniformidade das técnicas utilizadas é incompatível com a flexibilidade necessária à boa prática clínica. Em resumo, no que diz respeito à generalização, a crítica ao uso do ensaio clínico randomizado na pesquisa em psicoterapia é que ele testa um tratamento um tanto artificial utilizando clientes atípicos; assim, demonstrar que um tratamento é eficaz nessas circunstâncias não o faz necessariamente efetivo, isto é, útil no mundo real da prática clínica.

Em conclusão, a despeito de ser reprovado por diversos pesquisadores pelas razões apontadas acima (abranger apenas diagnósticos do DSM, impossibilidade de cegamento, dificuldade em padronizar a variável independente, concepção de placebo, composição dos grupos-controle, etc.), o ensaio clínico randomizado é um método de pesquisa que possibilita estabelecer relações de dependência com bastante precisão e, por isso, tem sido considerado o padrão-ouro para determinar a eficácia das diferentes modalidades de psicoterapia.

\section{EXPERIMENTO DE CASO ÚNICO}

No experimento de caso único, um mesmo indivíduo é submetido a todas as condições da pesquisa e, em vez de ter seu desempenho comparado ao de um grupo, ele serve como seu próprio controle. Isso não significa que apenas um único cliente seja utilizado, mas que seus dados são tratados individualmente (Sampaio et al., 2008).

A premissa do delineamento experimental de caso único é que a eficácia de uma intervenção pode ser estabelecida por meio da mensuração continuada do comportamento-alvo do indivíduo antes, durante e após introduzir e remover aquela intervenção. Para isso, o experimento é divido em duas condições genéricas - linha de base e tratamento. Na linha de base, a variável dependente (um ou mais comportamentos-alvo, escores em questionários de autorrelato, etc.) é mensurada repetidas vezes para obter-se o desempenho-padrão do indivíduo na ausência da intervenção, condição análoga ao grupo-controle do ensaio clínico randomizado. $\mathrm{Na}$ condição de tratamento, a variável independente (uma ou mais ações terapêuticas definidas operacionalmente) é introduzida e novas medidas da variável dependente são realiza- das (Barlow, Nock, \& Hersen, 2008; Hurst \& Nelson-Gray, 2006).

A fim de constatar se há uma relação de dependência entre as ações do terapeuta e as mudanças no cliente, é fundamental medir repetidamente a variável dependente sob uma mesma condição até se obter dados estáveis. Entretanto, como alertam Sampaio et al. (2008), estabilidade não significa imutabilidade, mas sim que o desempenho do participante tem pouca variação ou oscila dentro um padrão. Variações muito grandes (i.e., instabilidade) impossibilitam qualquer conclusão acerca do efeito da variável independente sobre a variável dependente, sendo que as mudanças observadas podem ser resultado de variáveis intervenientes à pesquisa (história de vida, maturação, etc.). Ademais, os mesmos autores lembram que a introdução da intervenção pode não produzir mudanças imediatas ou abruptas no desempenho do cliente, uma vez que a construção de um novo padrão comportamental costuma depender de diversas interações do indivíduo com o ambiente. Por essas razões, a estabilidade da variável dependente é o principal critério utilizado pelos pesquisadores para decidir se o experimento deve permanecer na fase em que se encontra ou prosseguir para a próxima condição - introdução ou remoção da variável independente (Sampaio et al., 2008).

Ademais, a relação de dependência observada entre as ações terapêuticas e as mudanças no comportamento do cliente deve ser sistematicamente replicada, o que pode ocorrer tanto intrassujeitos quanto entresujeitos. Para a replicação intrassujeitos, o delineamento com maior rigor experimental é o de reversão ou $A B A B$ (sendo que $A$ é a condição de linha de base e $B$ a condição de tratamento), que possibilita realizar sucessivas verificações dos efeitos da variável independente sobre a variável dependente por meio da introdução, retirada e reintrodução da variável independente. 0 delineamento de reversão pode variar quanto ao número e a ordem de fases (e.g., $A B A, B A B A)$ e também quanto à quantidade de variáveis manipuladas (e.g., ABACACADA, sendo que as letras $B, C$ e D representam diferentes intervenções; Barlow, Nock, \& Hersen, 2008; Hurst \& Nelson-Gray, 2006).

A replicação entresujeitos, por sua vez, pode ser obtida tanto pelo uso do mesmo delineamento de reversão com diferentes indivíduos assim como por meio do delineamento de linha de base múltipla, no qual diferentes linhas de base são estabelecidas para cada cliente e o procedimento terapêutico é introduzido em diferentes momentos para cada um deles. Dessa forma, não é necessário fazer reversões para demonstrar os efeitos da variável independente sobre a variável dependente, o que é especialmente relevante para avaliar o resultado de intervenções que são irreversíveis por motivos práticos ou éticos. Vale apontar que, além de ser utilizado para investigar o efeito de uma variável independente sobre o 


\section{WIINTERACÃO EM ET. PSICOLOGIA}

desempenho de dois ou mais participantes, o delineamento de linha de base múltipla também pode ser empregado para examinar o efeito de uma variável independente sobre dois ou mais comportamentos de um mesmo indivíduo ou, ainda, o efeito de uma variável independente sobre um mesmo comportamento de um mesmo indivíduo em duas ou mais condições ambientais (Barlow, Nock, \& Hersen, 2008; Hurst \& Nelson-Gray, 2006).

Além do $A B A B$ e da linha de base múltipla, outros delineamentos experimentais de caso único são: retirada, no qual o tratamento é interrompido para investigar seus efeitos ao longo do tempo; sonda, que avalia se a intervenção realizada teve algum efeito sobre um desempenho que não foi alvo da intervenção; e mudança de critério, em que a condição experimental é modificada toda vez que o desempenho do indivíduo alcança um critério previamente estabelecido (Barlow, Nock, \& Hersen, 2008; Hurst \& Nelson-Gray, 2006).

Tradicionalmente, os dados obtidos nos experimentos de caso único são dispostos em gráficos, de modo que diferenças no nível, tendência e variabilidade das medidas do desempenho do cliente são analisadas visualmente, e não estatisticamente, para determinar se houve e qual foi o efeito da intervenção em estudo. Em alguns casos, análises estatísticas podem complementar a interpretação dos resultados, sobretudo quando os dados não apresentam um padrão explícito ou quando há dúvidas sobre sua significância clínica (Barlow, Nock, \& Hersen, 2008; Hurst \& Nelson-Gray, 2006).

0 experimento de caso único tem sido defendido por diversos pesquisadores (e.g., Blampied, 1999; Hurst \& NelsonGray, 2006; Lundervold \& Belwood, 2000) como um método que deve ter papel central na produção de evidências em psicoterapia, por diversas razões: possibilita o estudo aprofundado do indivíduo em suas particularidades ao mesmo tempo em que mantém a objetividade e o rigor experimental necessários à ciência; facilita o desenvolvimento de intervenções eficazes para transtornos ou padrões comportamentais cuja baixa prevalência impede a criação de grupos amostrais; supera os problemas inerentes aos estudos de grupo de extrair um valor médio relativo ao desempenho de vários indivíduos, proporcionando a análise individualizada dos resultados da psicoterapia em questão; permite decompor os diferentes componentes de uma intervenção para comparar seus efeitos diferenciais; e contribui para inovação clínica de forma ética, na medida em que viabiliza a investigação cuidadosa de novos procedimentos terapêuticos sem que seja necessário expor um grande número de pessoas a uma intervenção sem sustentação empírica. No que diz respeito a esse último ponto, Hurst e Nelson-Gray (2006) explicam que a eficácia de determinada psicoterapia não requer obrigatoriamente pesquisas experimentais de grupo, podendo ser esta- belecida unicamente por meio de experimentos de caso único. Nessa direção, vale lembrar que a listagem de tratamentos empiricamente sustentados oferecida pela Divisão 12 da American Psychological Association (Chambless et al., 1998) contém terapêuticas que foram assim classificadas tendo por base somente experimentos de caso único.

Especialistas (e.g., Barlow, Nock, \& Hersen, 2008; Blampied, 1999; Hurst \& Nelson-Gray, 2006) em delineamento experimental de caso único alertam para algumas limitações desse método de pesquisa: em alguns casos, a reversão para a condição de linha de base é cientificamente inexequível, pois os efeitos da variável independente sobre a variável dependente são permanentes - por exemplo, não é possível remover a aprendizagem de certo repertório comportamental; em outros casos, a reversão é eticamente inaceitável, como retornar a uma condição em que ocorrem comportamentos danosos para o indivíduo; além disso, a demonstração cabal dos efeitos de uma intervenção depende de muitas replicações em diversos clientes, o que pode delongar a produção de evidências.

\section{ESTUDO DE CASO}

O estudo de caso é a narrativa fiel, sistemática e longitudinal de um processo terapêutico, geralmente baseado na memória do terapeuta, que abrange a história de vida do cliente, as intervenções realizadas, as mudanças observadas e outras informações relevantes, que possam levar a uma meIhor compreensão do caso atendido. Dessa forma, o estudo de caso não visa investigar o efeito de uma variável específica ou testar hipóteses preestabelecidas, mas descrever de forma cuidadosa e detalhada um processo terapêutico único em toda a sua complexidade (Serralta, Nunes, \& Eizirik, 2011; Silvares \& Banaco, 2000/2012; Stiles, 2006).

Ao longo da história da Psicologia clínica, o estudo de caso teve papel central no desenvolvimento das diferentes abordagens terapêuticas. Alguns exemplos famosos são: na psicanálise, Anna O. (Freud, 1893-1895/1957); na terapia comportamental, Pequeno Albert (Watson \& Rayner, 1920); na terapia centrada no cliente, Dibbs (Axline, 1964); etc.

Por se basear em dados naturalísticos da própria atividade clínica, o estudo de caso é defendido por diversos autores (Dattilio, 2006; Hill, 2006; Serralta et al., 2011; Stiles, 2006) como um meio essencial de produção de evidências empíricas, capaz de superar os limites impostos pelos métodos experimentais. Na perspectiva desses autores, a necessidade requerida pela pesquisa experimental de isolar o fenômeno de interesse e controlar variáveis tornaria o processo terapêutico artificial, obscureceria a apreensão de episódios clínicos relevantes, restringiria a expressão da subjetividade por 


\section{INTERACÃO EM LF PSICOLOGIA}

parte do cliente e impediria o exame de dados inesperados às hipóteses em investigação, a ponto de a pesquisa ficar à margem da prática clínica real. 0 estudo de caso, inversamente, reproduziria o contexto original da prática, levando em consideração qualquer fenômeno que surgir ao longo do processo terapêutico e as complexas relações entre variáveis, o que contribuiria para a diminuição da lacuna entre pesquisa e prática clínica. Assim, o estudo de caso não seria apenas útil, mas necessário (Dattilio, 2006; Stiles, 2006). Além disso, o estudo de caso é o método por excelência para a análise de condições raras. Nas palavras de Alvez-Mazzotti (2006):

[Uma] razão que justifica a opção por um estudo de caso é o fato de ele ser extremo ou único. Essa é a situação, comum em Psicologia Clínica, na qual uma pessoa, devido a uma deficiência ou a condições de vida não usuais, apresenta um padrão de comportamento extremamente raro e para o qual não existem ainda teorias explicativas convincentes. 0 fato de que tais situações são instigantes e extremamente raras justifica a análise em profundidade de qualquer caso em que ocorram tais padrões de comportamento. (p. 644)

Todavia, muitos pesquisadores (e.g., Reed, Kihlstrom, \& Messer, 2006) contestam o préstimo científico do estudo de caso por carecer de validade interna e de validade externa. Entre os problemas de validade interna estão a omissão ou distorção de informações relevantes devido ao esquecimento ou expectativas de sucesso e à pouca objetividade do julgamento tanto do terapeuta quanto do cliente para determinar os resultados da intervenção. No que tange à validade externa, o estudo de caso possui duas fraquezas muito importantes: impossibilidade de replicação direta e dificuldade de generalização dos resultados, uma vez que a descrição envolve aspectos tão individualizados que tornam os dados incomparáveis com os de outros indivíduos; dificuldade de combinar resultados de diferentes estudos para derivar uma conclusão sobre eficácia, sobretudo porque não foram desenvolvidos métodos específicos de revisão de literatura e de meta-análise para estudos de caso.

Em razão dessas limitações, Kazdin (1981) afirma que "estudos de caso nunca podem substituir a experimentação" (p. 183). Em consonância, Cassas e Regis Neto (2010) sugerem que o estudo de caso deveria ser empregado apenas no estágio inicial da investigação científica de determinado fenômeno, como o ponto de partida para a elaboração de pesquisas mais rigorosas. Nesse contexto, os autores propõem uma distinção entre o estudo de caso propriamente dito e o relato de caso, este concebido como uma forma didática de ilustrar e disseminar o conhecimento já produzido para os profissionais em formação - portanto, sem valor de evidência científica para a sustentação de uma prática terapêutica.
Com o intuito de tornar o estudo de caso mais relevante para a produção de evidências empíricas em psicoterapia, Kazdin (1981) elenca cinco procedimentos que podem ser utilizados para aprimorar sua validade e confiabilidade: coleta de dados quantitativos ao invés de qualitativos, o que é necessário para medir objetivamente as mudanças ocorridas e tornar o estudo replicável; avaliação contínua das variáveis dependentes e não apenas pré e pós-intervenção, o que garante que as mudanças observadas não sejam produto de outras variáveis, tais como características da coleta e da análise dos dados (e.g., exposição ao instrumento de medida de resultado, regressão para a média, etc.); conhecimento da estabilidade do problema clínico (que pode provir tanto da avaliação contínua ao longo do processo terapêutico quanto de pesquisas que demonstram se determinado problema clínico tem maior probabilidade de melhorar, piorar ou permanecer o mesmo ao longo do tempo), pois, se o comportamento-alvo está estável e a mudança coincide com a intervenção, então é possível inferir uma relação de dependência; observação de efeitos imediatos e acentuados, que indicam que a intervenção é a responsável pelas mudanças, ao invés de demorados e brandos, que sugerem que elas podem ser produto de fatores externos (história de vida, maturação, etc.); inclusão de múltiplos casos, o que pode confirmar ou não a demonstração de uma relação de dependência entre as ações do terapeuta e as mudanças do cliente.

Desde que Kazdin (1981) fez tais recomendações há mais de 30 anos, a preocupação com o rigor dos estudos de caso aumentou. Por exemplo, na política editorial do periódico Psychotherapy, três diretrizes são fornecidas para a elaboração de um estudo de caso: aplicação de duas medidas de resultado padronizadas e de uma medida de processo terapêutico em três momentos distintos no tempo (e.g., antes, durante e depois da psicoterapia); apresentação da significância clínica dos resultados (ou seja, se os resultados ocasionados pela intervenção têm um impacto palpável sobre o funcionamento do cliente em seu cotidiano; e.g., Ogles, Lunnen, \& Bonesteel, 2001); transcrição de trechos do atendimento que destaquem as principais intervenções realizadas e os mecanismos de mudança envolvidos.

Em suma, embora os estudos de caso sejam profícuos para examinar casos raros e para iniciar a pesquisa científica sobre determinado fenômeno clínico, há algum consenso de que o método é bastante limitado para o estabelecimento de relações de dependência, fundamental para o entendimento dos efeitos de uma intervenção (Dattilio, 2006; Hill, 2006; Messer, 2007; Silvares \& Banaco, 2000/2012; Stiles, 2006). De qualquer forma, os estudos de caso podem ser relevantes para a produção de conhecimento em psicoterapia se forem adotados procedimentos (como os elencados por Kazdin em 


\section{INTERACÃO EM * PSICOLOGIA}

1981 e, mais recentemente, pela política editorial de Psychotherapy) que fortaleçam sua qualidade científica.

\section{REVISÕES DE LITERATURA E DIRETRIZES CLIINICAS}

Um fator que pode dificultar o uso de evidências é que raramente um único estudo, por melhor que ele tenha sido conduzido, é capaz de fornecer uma prova definitiva do efeito de determinada terapêutica, na medida em que diferentes estudos podem chegar a resultados conflitantes e até opostos, seja devido a características dos participantes, divergências metodológicas, heterogeneidade no modo de analisar e interpretar os dados, entre outras razões. Dessa forma, fica difícil saber quais evidências são mais ou menos confiáveis e, portanto, quais devem ou não sustentar a prática clínica. Tal problema pode ser solucionado por meio da revisão de literatura, um método de pesquisa que tem por objetivo analisar comparativamente os resultados de um grande número de estudos e, assim, prover uma síntese dos efeitos de certa intervenção (Kaczorowski, 2009). Duas modalidades de revisão de literatura costumam ser utilizadas no contexto da prática baseada em evidências em Psicologia: a revisão sistemática (com ou sem meta-análise) e a revisão integrativa.

A revisão sistemática consiste num protocolo rigoroso e replicável que orienta a identificação, avaliação e síntese de pesquisas clínicas sobre determinada intervenção. A elaboração de uma revisão sistemática geralmente usa o formato PI$\mathrm{CO}$, acrograma para população, intervenção, comparação e resultado (do inglês, outcome), como, por exemplo, "qual é a eficácia da terapia cognitivo-comportamental para indivíduos adultos com transtorno depressivo maior em comparação com a fluoxetina?" (Kaczorowski, 2009). Atualmente, a maioria das revisões sistemáticas em psicoterapia inclui metaanálise, um procedimento estatístico utilizado para calcular a magnitude de efeito da intervenção, isto é, quanto de mudança ocorreu na variável dependente (e.g., sintomas de depressão) após a exposição à variável independente (e.g., terapia cognitivo-comportamental). Para isso, o tamanho do efeito de cada um dos estudos é calculado e, posteriormente, os valores individuais são agrupados por meio de diversos procedimentos matemáticos, o que permite determinar se a mudança promovida pela intervenção é clinicamente significativa (Field, 2013).

Por sua vez, a revisão integrativa tem os mesmos objetivos da revisão sistemática, mas é menos rigorosa no que se refere aos delineamentos empregados, abrangendo pesquisas experimentais (e.g., ensaios clínicos randomizados) e não experimentais (e.g., estudos qualitativos), incluindo na análise todos os estudos que foram encontrados. Assim, a revisão integrativa é o método mais apropriado para analisar as evidências de determinada intervenção quando a quantidade e/ou a qualidade dos estudos não permitem a execução de uma revisão sistemática. Ao mesmo tempo, tais características impõem limitações na confiabilidade da revisão integrativa, uma vez que não foram desenvolvidos protocolos que orientem como devem ser agrupados os dados produzidos por diferentes métodos de pesquisa ou qual peso deve ser atribuído a eles a depender do tipo de delineamento empregado e de sua qualidade metodológica (Souza, Silva, \& CarvaIho, 2010).

É importante observar que a síntese das evidências de eficácia de uma modalidade de psicoterapia na revisão de literatura requer uma avaliação crítica da qualidade metodológica dos estudos revisados, que geralmente ocorre por meio do preenchimento de um checklist padronizado que estima a validade e a confiabilidade dos dados de modo a contrabalancear a expressividade de cada um deles no conjunto total das evidências. A escolha de um instrumento adequado de avaliação crítica é parte fundamental da prática baseada em evidências, uma vez que a informação sobre a validade e a confiabilidade é extremamente importante para que os consumidores de pesquisa sejam capazes de determinar se as evidências são relevantes e, portanto, se devem ou não ser transpostas para os mais diversos âmbitos, tais como prática clínica, formação de profissionais, formulação de políticas públicas, etc. (Katrak, Bialocerkowski, Massy-Westropp, Kumar, \& Grimmer, 2004).

Existem diversos instrumentos para avaliar a qualidade metodológica de ensaios clínicos randomizados (e.g., Katrak et al., 2004) e, mais recentemente, em um esforço para que dados oriundos de experimentos de caso único fossem incorporados no contexto da prática baseada em evidências, o What Works Clearinghouse, um núcleo de pesquisa do Departamento de Educação dos EUA, desenvolveu critérios de avaliação da qualidade metodológica de experimentos de caso único (e.g., Kratochwill et al., 2013).

Por fim, a síntese de evidências em revisões sistemáticas culmina na elaboração de diretrizes clínicas, que são recomendações específicas de determinadas intervenções baseadas numa avaliação dos benefícios e danos das diferentes alternativas que existem (e.g., Woolf, Grol, Hutchinson, Eccles, \& Grimshaw, 1999). Atualmente, as diretrizes clínicas costumam se basear no Grading of Recommendations Assessment, Development and Evaluation ${ }^{2}$ (GRADE), um sistema que avalia a qualidade das evidências e a força das recomendações em saúde por meio de diversos fatores, como o delineamento dos estudos, a qualidade metodológica, a variabilidade dos resultados, o balanço entre os efeitos desejáveis e indesejáveis da intervenção, etc. 


\section{INTERACÃO EM LF PSICOLOGIA}

\section{CONSIDERAC̃̃ES SOBRE OS MÉTODOS DE PESQUISA PARA A PRODUÇÃO D̉E EVIDÊNCIAS}

Em um debate sobre os pontos fortes e fracos dos diferentes métodos de produção de evidências em psicoterapia (Stiles et al., 2006), pesquisadores compartilham da opinião de que o progresso da pesquisa clínica requer múltiplas perspectivas metodológicas e apontam dois motivos que justificam a integração de ensaios clínicos randomizados, experimentos de caso único e estudos de caso qualitativos. Primeiro, o grau de confiança em um dado empírico é maior quando métodos diferentes produzem resultados semelhantes. Segundo, o uso de métodos com características distintas entre si pode maximizar a variabilidade das descobertas.

Há consenso, também, de que os estudos de caso qualitativos são úteis para a inovação clínica, constituindo um primeiro passo na identificação de procedimentos potencialmente eficazes. Nesse sentido, dados de estudos qualitativos podem servir como ponto de partida para o levantamento de hipóteses que serão verificadas em pesquisas experimentais. Além disso, quando já existem dados experimentais que comprovam a eficácia de determinados pacotes de tratamento, os estudos de casos podem servir como uma ferramenta importante para a individualização dos procedimentos e para indicar a investigação dos mecanismos de mudança. Todavia, embora o método qualitativo permita compreender a vivência subjetiva do cliente em sua complexidade, o estudo de caso é um método deficiente para a produção de evidências porque carece das duas características centrais da pesquisa científica - validade interna e validade externa. Conforme explicitado anteriormente, tal limitação pode ser parcialmente superada aderindo os critérios de Kazdin (1981) e da política editorial de Psychotherapy, mas não suprem a necessidade de experimentação.

A pesquisa experimental é vital por ser a única forma possível de atingir o objetivo final da pesquisa em psicoterapia, que é provar que determinada terapêutica gera melhoras em certo quadro clínico. Dessa forma, tanto os ensaios clínicos randomizados quanto os experimentos de caso único são necessários para a produção de evidências. Vale observar que, no debate supracitado (Stiles et al., 2006), Steven D. Hollon, um ferrenho defensor do ensaio clínico randomizado, afirma "eu os considero [experimentos de caso único] como o equivalente lógico dos ensaios clínicos randomizados e reconheço que eles conferem algumas vantagens práticas sobre os delineamentos com grupos grandes" (p. 117). Enquanto que os ensaios clínicos randomizados têm como grande vantagem o estabelecimento de relações de dependência em grupos representativos de uma população, os experimentos de caso único têm, a um só tempo, o rigor experimental de um ensaio clínico randomizado e a perscruta de um estudo de caso, embora ao custo de pouca representatividade. Enfim, ambos os métodos são relevantes para a produção de evidências empíricas em psicoterapia porque permitem testar com alguma exatidão os efeitos de uma intervenção.

Com base no que foi apresentado, é possível imaginar que o desenvolvimento de procedimentos terapêuticos eficazes obedeceria a uma sequência de estágios. Inicialmente, uma nova terapêutica seria concebida a partir de observações clínicas, pesquisa básica ou raciocínio teórico e, então, investigada de modo exploratório em estudos de caso. Posteriormente, os procedimentos seriam testados com mais precisão em experimentos de caso único, que indicariam ou não a necessidade de pesquisá-los numa amostra maior em um ensaio clínico randomizado. o próximo passo seria avaliar se as intervenções que se mostraram eficazes (i.e., que apresentaram resultados positivos em condições de pesquisa) são, de fato, efetivas (i.e., têm resultados positivos no contexto real da prestação de serviços). Por último, diferentes estudos individuais de eficácia e de efetividade deveriam ser sumarizados em revisões de literatura em vista a estabelecer de modo mais conclusivo os efeitos de determinada modalidade de intervenção psicoterápica.

Ademais, uma vez que determinada terapêutica teve sua eficácia comprovada em estudos experimentais, faz-se necessário avançar para a identificação dos mecanismos de mudança que explicam como ela produz melhora, o que é viabilizado pela pesquisa de processo. Dito de outra forma, ensaios clínicos randomizados e experimentos de caso único podem estabelecer com precisão a relação de dependência entre uma intervenção e a mudança do quadro clínico, mas não explica o porquê dessa relação. Por exemplo, depois que diversos estudos demonstraram que a dessensibilização sistemática é eficaz para curar fobias específicas, pesquisadores desmantelaram esse procedimento em pesquisas de processo e concluíram que o mecanismo comportamental responsável pelos seus efeitos era a extinção respondente e não o contracondicionamento por inibição recíproca, como originalmente hipotetizado (Marks, 1975). Segundo Kazdin (2001, 2009), conhecer os mecanismos de mudança é importante por, pelo menos, duas razões: as diversas modalidades de terapia seriam melhor organizadas caso fosse constatado que atuam por meio dos mesmos processos e otimizaria a qualidade da intervenção, pois apenas os procedimentos críticos seriam utilizados. Vale notar que tais razões se tornam ainda mais importantes quando se leva em consideração que as diferentes terapias geralmente são constituídas de um grande "pacote" que abrange uma miríade de técnicas. Porém, apesar de sua extrema relevância, muito pouco é sabido atualmente sobre os mecanismos de mudança subjacentes às terapêuticas com eficácia comprovada, o que coloca a 


\section{W INERACÄOEM ET PSICOLOGIA}

pesquisa de processo no centro das atenções no cenário atual.

Em síntese, o presente artigo apresentou os pontos fortes e fracos dos diferentes métodos de pesquisa empírica que existem para o estabelecimento de eficácia das psicoterapias. Espera-se que ele contribua para a formação de pesquisadores clínicos brasileiros e, por conseguinte, favoreça uma maior participação da Psicologia brasileira no movimento da prática baseada em evidências em Psicologia.

\section{DECLARAÇÃO DE CONFLITOS DE INTERESSES}

O autor declara que não há conflitos de interesses no presente artigo.

\section{REFERÊNCIAS}

Alvez-Mazzotti, A. D. (2006). Usos e abusos dos estudos de caso. Cadernos de Pesquisa, 36, 637-651.

American Psychiatric Association. (2013). Diagnostic and statistical manual of mental disorders: DSM-5. Arlington: American Psychiatric Publishing.

American Psychological Association. (2006). Evidence-based practice in psychology: APA presidential task force on evidence-based practice. American Psychologist, 61, 271285. http://dx.doi.org/10.1037/0003-066X.61.4.271

Axline, V. (1964). Dibs in search of self. New York: Ballatine Books.

Barlow, D. H., Nock, M. K., \& Hersen, M. (2008). Single case experimental designs: Strategies for studying behavior change (3a ed.). Boston: Allyn and Bacon.

Baskin, T. W., Tiemey, S. C., Minami, T., \& Wampold, B. E. (2003). Establishing specificity in psychotherapy: A metaanalysis of structural equivalence of placebo controls. Journal of Consulting and Clinical Psychology, 71, 973-979. http://dx.doi.org/10.1037/0022-006X.71.6.973

Blampied, N. M. (1999). A legacy neglected: Restating the case for single-case research in cognitive-behaviour therapy. Behaviour Change, 16, 89-104. http://dx.doi.org/10.1375/bech.16.2.89

Borkovec, T. D., \& Castonguay, L. G. (2006). Effectiveness research. In J. C. Norcross, L. E. Beutler, \& R. F. Levant (Orgs.), Evidence-based practices in mental health: Debate and dialogue on the fundamental questions (pp. 89-96). Washington: American Psychological Association.

Cassas, F. A., \& Regis Neto, D. M. (2010). Estudos de caso e relatos de caso: Uma reflexão inicial sobre suas possíveis diferenças e funções. Em M. R. Garcia, P. R. Abreu, E. N. P. Cillo, P. B. Faleiros, \& P. Piazon (Orgs.), Sobre comporta- mento e cognição: Terapia comportamental e cognitiva (Vol. 27, pp. 280-285). Santo André: Esetec.

Chambless, D. L., Baker, M., Baucom, D. H., Beutler L. E, CaIhoun K. S., Crits-Christoph, P., . . Woody, S. R. (1998). Update on empirically validated therapies, II. The Clinical Psychologist, 51, 3-16.

Dattilio, F. M. (2006). Does the case study have a future in the psychiatric literature? International Journal of Psychiatry in Clinical Practice, 10, 195-203. http://dx.doi.org/10.1080/13651500600649895

Field, A. P. (2013). Meta-analysis in clinical psychology research. In J. S. Comer, \& P. C. Kendall (Orgs.), The Oxford handbook of research strategies for clinical psychology (pp. 317-335). New York: Oxford University Press.

Freud, S. (1957). Studies on hysteria. In J. Strachey (Org. \& Trans.), The standard edition of the complete psychological works of Sigmund Freud (Vol. 2). London: Hogarth Press. (Trabalho original publicado em 1893-1895)

Hill, C. E. (2006). Qualitative research. In J. C. Norcross, L. E. Beutler, \& R. F. Levant (Orgs.), Evidence-based practices in mental health: Debate and dialogue on the fundamental questions (pp. 74-81). Washington: American Psychological Association.

Hill, C., Sim, W., Spangler, P., Stahl, J., Sullivan, C. \& Teyber, E. (2008). Therapist immediacy in brief psychotherapy: Case study II. Psychotherapy: Theory, Research, Practice, Training, 45, 298-315. http://dx.doi.org/10.1037/a0013305

Hollon, S. D. (2006). Randomized clinical trials. In J. C. Norcross, L. E. Beutler, \& R. F. Levant (Orgs.), Evidence-based practices in mental health: Debate and dialogue on the fundamental questions (pp. 96-105). Washington: American Psychological Association.

Hurst, R. M., \& Nelson-Gray, R. (2006). Single-participant (S-P) design research. In J. C. Norcross, L. E. Beutler, \& R. F. Levant (Orgs.), Evidence-based practices in mental health: Debate and dialogue on the fundamental questions (pp. 6473). Washington: American Psychological Association.

Kaczorowski, J. (2009). Standing on the shoulders of giants: Introduction to systematic reviews and meta-analyses. Canadian Family Physician, 55, 1155-1156.

Katrak, P., Bialocerkowski, A. E., Massy-Westropp, N. Kumar, V. S. S., \& Grimmer, K. A. (2004). A systematic review of the content of critical appraisal tools. BMC Medical Research Methodology, 4, 1-11. http://dx.doi.org/10.1186/1471-2288-4-22

Kazdin, A. E. (1981). Drawing valid inferences from case studies. Journal of Consulting and Clinical Psychology, 49, 183-192. http://dx.doi.org/10.1037/0022-006X.49.2.183

Kazdin, A. E. (1992). Research design in clinical psychology (2a ed.). Needham Heights: Allyn and Bacon. 


\section{W NTERAC̄OOEM ET. PSICOLOGIA}

Kazdin, A. E. (2001). Progression of therapy research and clinical application of treatment require better understanding of the change process. Clinical Psychology: Science and Practice, 8, http://dx.doi.org/10.1093/clipsy/8.2.143

Kazdin, A. E. (2009). Understanding how and why psychotherapy leads to change. Psychotherapy Research, 19, 418428. http://dx.doi.org/10.1080/10503300802448899

Kendall, J. M. (2003). Designing a research project: Randomised controlled trials and their principles. Emergency Medicine Journal, 20, 164-168. http://dx.doi.org/10.1136/emj.20.2.164

Kendall, P. C., Comer, J. S., \& Chow, C. (2013). The randomized controlled trial: Basics and beyond. In J. S. Comer \& P. C. Kendall (Orgs.), The Oxford handbook of research strategies for clinical psychology (pp. 40-61). New York: Oxford University Press.

Kihlstrom, J. F. (2006). Scientific research. In J. C. Norcross, L. E. Beutler, \& R. F. Levant (Orgs.), Evidence-based practices in mental health: Debate and dialogue on the fundamental questions (pp. 23-31). Washington: American Psychological Association.

Kratochwill, T. R., Hitchcock, J. H., Horner, R. H., Levin, J. R., Odom, S. L., Rindskopf, D. M., \& Shadish, W. R. (2013). Single-case intervention research design standards. Remedial and Special Education, 34, 26-38.

Leonardi, J. L., \& Meyer, S. B. (2015). Prática baseada em evidências em psicologia e a história da busca pelas provas empíricas da eficácia das psicoterapias. Psicologia: Ciência e Profissão, 35, 1139-1156. http://dx.doi.org/10.1590/1982-3703001552014

Lundervold, D. A., \& Belwood, M. F. (2000). The best kept secret in counseling: Single-case $(N=1)$ experimental designs. Journal of Counseling \& Development, 78, 92-102. http://dx.doi.org/10.1002/j.1556-6676.2000.tb02565.x

Marks, I. (1975). Behavioral treatments of phobic and obsessive-compulsive disorders: A critical appraisal. In M. Hersen, R. M. Eisler, \& P. M. Miller (Orgs.), Progress in behavior modification (Vol. 1, pp. 66-158). New York: Academic Press.

Messer, S. B. (2007). Psychoanalytic case studies and the pragmatic case study method. Pragmatic Case Studies in Psychotherapy, 3 , 55-58. http://dx.doi.org/10.14713/pcsp.v3i1.894

Nathan, P. E., Stuart, S. P., \& Dolan, S. L. (2000). Research on psychotherapy efficacy and effectiveness: Between Scylla and Charybdis? Psychological Bulletin, 126, 964-981. http://dx.doi.org/10.1037/0033-2909.126.6.964

Norcross, J. C., Beutler, L. E., \& Levant, R. F. (Orgs.). (2006). Evidence-based practice in mental health: Debate and dialogue on the fundamental questions. Washington: American Psychological Association.
Ogles, B. M., Lunnen, K. M., \& Bonesteel, K. (2001). Clinical significance: History, application, and current practice. Clinical Psychology Review, 21, 421-446. http://dx.doi.org/10.1016/S0272-7358(99)00058-6

Persons, J. B., \& Silberschatz, G. (2003). Are results of randomized controlled trials useful to psychotherapists? In A. E. Kazdin (Org.), Methodological issues \& strategies in clinical research (3a ed., pp. 547-568). Washington: American Psychological Association.

Reed, G. M., Kihlstrom, J. F., \& Messer, S. B. (2006). What qualifies as evidence of effective practice? In J. C. Norcross, L. E. Beutler, \& R. F. Levant (Orgs.), Evidence-based practices in mental health: Debate and dialogue on the fundamental questions (pp. 13-55). Washington: American Psychological Association.

Rosenthal, D., \& Frank, J. D. (1956). Psychotherapy and the placebo effect. Psychological Bulletin, 53, 294-302. http://dx.doi.org/10.1037/h0044068

Sampaio, A. A. S., Azevedo, F. H. B., Cardoso, L. R. D., Lima, C., Pereira, M. B. R., \& Andery, M. A. P. A. (2008). Uma introdução aos delineamentos experimentais de sujeito único. Interação em Psicologia, 12, 151-164. http://dx.doi.org/10.5380/psi.v12i1.9537

Serralta, F. B., Nunes, M. L. T., \& Eizirik, C. L. (2011). Considerações metodológicas sobre o estudo de caso na pesquisa em psicoterapia. Estudos de Psicologia (Campinas), 28, 502-510. http://dx.doi.org/10.1590/S0103-166X2011000400010

Silvares, E. F. M., \& Banaco, R. A. (2012). O estudo de caso clínico comportamental. Em E. F. M. Silvares (Org.). Estudos de caso em psicologia clínica comportamental infantil: Vol. 1. Fundamentos conceituais, estudos grupais e estudos relativos a problemas de saúde (7a ed., pp. 31-48). Campinas: Papirus. (Trabalho original publicado em 2000)

Souza, M. T., Silva, M. D., \& Carvalho, R. (2010). Revisão integrativa: 0 que é e como fazer. Einstein, 8, 102-106. http://dx.doi.org/10.1590/s1679-45082010rw1134

Starcevic, V. (2003). Psychotherapy in the era of evidence-based medicine. Australasian Psychiatry, 11, 278-281. http://dx.doi.org/10.1046/j.1440-1665.2003.00575.x

Stiles, W. B. (2006). Case studies. In J. C. Norcross, L. E. Beutler, \& R. F. Levant (Orgs.), Evidence-based practices in mental health: Debate and dialogue on the fundamental questions (pp. 57-64). Washington: American Psychological Association.

Stiles, W. B., Hurst, R. M., Nelson-Gray, R., Hill, C. E., Greenberg, L. S., Watson, J. C., . . Hollon, S. D. (2006). What qualifies as research on which to judge effective practice? In J. C. Norcross, L. E. Beutler, \& R. F. Levant (Orgs.), Evidence-based practices in mental health: Debate and dialogue on the fundamental questions (pp. 57-130). Washington: American Psychological Association. 


\section{WIIITERACÃO EM ET PSICOLOGIA}

Watson, J. B., \& Rayner, R. (1920). Conditioned emotional reactions. Journal of Experimental Psychology, 3, 1-14. http://dx.doi.org/10.1037/h0069608

Woolf, S. H., Grol, R., Hutchinson, A., Eccles, M., \& Grimshaw, J. (1999). Clinical guidelines: Potential benefits, limitations, and harms of clinical guidelines. BMJ: British Medical Journal, 318, 527-530.

${ }^{1}$ Neste contexto, é importante distinguir o conceito de eficácia do conceito de efetividade. Na literatura de prática baseada em evidências, eficácia diz respeito à obtenção de resultados clinicamente relevantes em condições controladas de pesquisa, enquanto que efetividade se refere à obtenção de tais resultados no âmbito da prestação de serviços (Borkovec \& Castonguay, 2006).

${ }^{2}$ Para o leitor interessado, o website do GRADE - www.gradeworkinggroup.org - lista um conjunto de publicações sobre como entendê-lo e utilizá-lo. 